Article

\title{
Gold/QDs-Embedded-Ceria Nanoparticles: Optical Fluorescence Enhancement as a Quenching Sensor
}

\author{
Nader Shehata $1,2,3,4,5, * \mathbb{D}$, Effat Samir ${ }^{6, *}$ and Ishac Kandas ${ }^{1,2,3}$ \\ 1 Center of Smart Nanotechnology and Photonics (CSNP), SmartCI Research Center, Alexandria University, \\ Alexandria 21544,Egypt; ishac@vt.edu \\ 2 Department of Engineering Mathematics and Physics, Faculty of Engineering, Alexandria University, \\ Alexandria 21544, Egypt \\ 3 Kuwait College of Science and Technology, Doha Area, 7th Ring Road, Safat 13133, Kuwait \\ 4 USTAR Bio-Innovation center, Utah State University, Logan, UT 84341, USA \\ 5 The Bradley Department of Electrical and Computer Engineering, Virginia Tech, Blacksburg, VA 24061, USA \\ 6 Department of Electrical Engineering, Old Dominion University, Norfolk, VA 23529, USA \\ * $\quad$ Correspondence: nader83@vt.edu (N.S.); effat_samir@mena.vt.edu (E.S.); Tel.: +20-10-9116-5300 (N.S.)
}

Received: 6 November 2019; Accepted: 3 January 2020; Published: 12 February 2020

\begin{abstract}
This work focuses on improving the fluorescence intensity of cerium oxide (ceria) nanoparticles (NPs) through added plasmonic nanostructures. Ceria nanoparticles are fluorescent nanostructures which can emit visible fluorescence emissions under violet excitation. Here, we investigated different added plasmonic nanostructures, such as gold nanoparticles (Au NPs) and Cadmium sulfide/selenide quantum dots (CdS/CdSe QDs), to check the enhancement of fluorescence intensity emissions caused by ceria NPs. Different plasmonic resonances of both aforementioned nanostructures have been selected to develop optical coupling with both fluorescence excitation and emission wavelengths of ceria. In addition, different additions whether in-situ or post-synthesis have been investigated. We found that in-situ Au NPs of a $530 \mathrm{~nm}$ plasmonic resonance wavelength provides the highest fluorescence emissions of ceria NPs compared to other embedded plasmonic structures. In addition to the optical coupling between plasmonic resonance of $\mathrm{Au}$ with the visible emissions fluorescence spectrum of ceria nanoparticles, the $530 \mathrm{~nm}$ in-situ Au NPs were found to reduce the bandgap of ceria NPs. We suggest that the formation of more tri-valent cerium ions traps energy levels along with more associated oxygen vacancies, which is responsible for increasing the fluorescence visible emissions intensity caused by ceria. As an application, the gold-ceria NPs is shown to optically detect the varied concentration of iron tiny particles in aqueous medium based on a fluorescence quenching mechanism. This work is promising in different applications such as biomarkers, cancer treatments, and environmental pollution monitoring.
\end{abstract}

Keywords: ceria nanoparticles; gold nanoparticles; quantum dots; fluorescence

\section{Introduction}

Cerium oxide (ceria) NPs can be considered to be one of the most promising nanostructures developed in the last two decades due to its oxide storage capacity based on reduction-oxidation (redox) characteristics [1]. The formed oxygen vacancies inside the crystalline structure of ceria are adsorbing centers for oxygen, radicals, and charged tiny metallic particles. Such vacancies are associated with the formation of tri-valent cerium ions trap energy levels, which are optically active through a reduced bandgap that is close to $3 \mathrm{eV}$. In addition, such tri-valent ions are responsible for visible fluorescence emissions when excited through violet or near ultra-violet (UV) exposure and 5d-4f energy levels transitions [2-5]. Hence, both the oxygen vacancies and optical fluorescence characteristics of ceria lead to using the optical nanostructures in wide variety of applications such as optical sensors for 
water quality monitoring, biomarkers, and free radical scavengers in cancer treatments [6-8]. However, there is still a lack of study regarding how to improve the optical fluorescence emissions of ceria NPs. In this work, the fluorescence emissions of ceria nanoparticles can be enhanced by using different plasmonic nanostructures, due to their importance in different applications, including solid oxide fuel cells and electro-catalysis [9-11]. Our idea was to test the impact of optical coupling between plasmonic nanostructures and ceria NPs in both excitation and emissions spectra. Here, we studied the impact of different plasmonic nanostructures such as Au NPs, cadmium sulfide/zinc sulfide (CdS/ZnS), and cadmium selenide/zinc sulfide (CdSe/ZnS) quantum dots (QDs) on the fluorescence emissions intensity of ceria NPs. Au NPs, CdS/ZnS, and CdSe/ZnS QDs were selected with specific diameters to have resonance wavelengths matched with both the excitation and emissions wavelengths of ceria NPs, which were around $430 \mathrm{~nm}$ and $530 \mathrm{~nm}$, respectively. Both plasmonic nanostructures have been added to ceria NPs whether in-situ, meaning within the initial precursors of the chemical precipitation synthesis process of ceria NPs, or post-treatment, which means to be added externally after the full preparation of ceria NPs.

Then, the idea of our work was to check the best embedded plasmonic nanostructure along with its condition, in-situ or post-treatment, to determine the enhanced fluorescence intensity of ceria NPs. The impact of the best plasmonic nanostructure, from the fluorescence intensity perspective, on ceria NPs was studied through the absorbance dispersion and correlated bandgap calculations. Then, the fluorescence intensity was detected according to the added plasmonic nanostructures. Then, as an application, the best optical plasmonic-embedded-ceria NPs were selected as optical sensing materials for metallic tiny particles, such as iron, in aqueous media. This application is important for water pollution monitoring using an optical quenching technique rather than a traditional electrochemical one, which suffers from reference electrode fouling $[12,13]$. Based on intensity quenching, the Stern-Volmer constant of the optimum selected plasmonic-ceria NPs, which indicates the sensitivity of quenching sensing, has been calculated to optically detect the added iron quenchers [14].

\section{Materials and Methods}

\subsection{Synthesis}

Ceria NPs were synthesized using a chemical precipitation technique for both relatively low-cost precursors and simplicity [15]. In detail, cerium chloride (Sigma Aldrich, St. Louis, MO, USA) of $0.5 \mathrm{~g}$ was added into $40 \mathrm{~mL}$ of distilled water. Then, the solution was stirred for two hours at a rotation rate of $500 \mathrm{rpm}$ and a temperature of $50^{\circ} \mathrm{C}$ for two hours at a stirring speed of $500 \mathrm{rpm}$. At the beginning of stirring, $1.6 \mathrm{~mL}$ of ammonium hydroxide (ammonia) was added as a catalyst. After two hours of warm stirring, the solution was stirred at the same rotation speed at room temperature overnight. This long stirring process helped to break most of formed ceria nanorods into NPs. Moreover, the first warming stage helped in converting cerium hydroxide into non-stoichiometric ceria NPs.

The used plasmonic nanostructures were Au NPs, CdS/ZnS, and CdSe/ZnSQDs. Au NPs (Sigma Aldrich, St. Louis, MO, USA) were selected with a diameter of $20 \mathrm{~nm}$ to have plasmonic resonance wavelength overlapped with a fluorescence emissions wavelength of $530 \mathrm{~nm}$. CdS/ZnS QDs (Sigma Aldrich, St. Louis, MO, USA) was selected to have a resonance overlap close to $430 \mathrm{~nm}$, and CdSe/ZnS QDs (Sigma Aldrich, St. Louis, MO, USA) with a resonance close to $530 \mathrm{~nm}$. We added all plasmonic nanoparticles with a weight concentration ratio of 1:3 compared to the solution of ceria nanoparticles. The different plasmonic nanostructures were added with two possibilities: in-situ which means to be added with the initial precursors stage; or post-treatment, which was to be stirred with ceria NPs after being synthesized. 


\subsection{Materials Characterization}

\subsubsection{Optical Characterizations}

Different samples of ceria NPs, whether they were pure or had embedded plasmonic nanostructures in-situ/post-added, were optically characterized. The synthesized plasmonic-ceria NPs were characterized based on both their fluorescence emissions intensity and their lifetime using a hand-made fluorescence spectroscopy setup. In this setup, the solution of plasmonic-ceria NPs was exposed to violet excitation from a laser module (Thorlab) with a relatively-narrow emissions spectrum centered at $430 \mathrm{~nm}$. Then, the fluorescent emissions intensity from the synthesized NPs was collected through a perpendicular Newport Cornerstone 130 monochromator for minimum scattering and scanned over the visible spectrum. Then, the detected signal which comes from the monochromator was detected using optical power meter (Newport, 1918R) after being amplified by an Oriel photomultiplier tube (PMT Newport 77360). The amplified emissions intensity was detected by a Newport Power meter 1918R. Based on the emissions intensity of the different plasmonic-ceria NPs, the samples with the highest emissions intensity were characterized through absorbance dispersion measurements using a dual-beam UV-Vis-NIR spectrometer (PG 90+ spectroscopy) over a spectrum between $300-700 \mathrm{~nm}$. Then, a direct bandgap could be calculated from the Urbach-tail results of the absorbance dispersion spectrum. This was done to detect the impact of added plasmonic nanostructures on the bandgap of ceria NPs.

\subsubsection{Morphological Analysis}

Images of some plasmonic-ceria NPs were obtained using a transmission electron microscope (TEM) (JEOL, Tokyo, Japan) with an accelerating potential of $80 \mathrm{KV}$. X-ray Diffraction (XRD) analysis of the synthesized NPs was measured using a Rigaku x-ray diffraction via $\mathrm{Cu} K \alpha$ radiation $(\lambda=+0.145 \mathrm{~nm})$.

\subsection{Optical Sensing Application}

As an application, different concentrations of iron tiny particles were added within the solution with the best fluorescence intensity. The fluorescence intensity quenching was measured using the fluorescence intensity setup. Also, fluorescence lifetime was detected for in-situ gold-ceria NPs at $530 \mathrm{~nm}$ for different iron particle concentrations. The used setup was a modified version of the previously mentioned fluorescence intensity setup with an added optical chopper step (MC2000B, Thorlab) and a chopping frequency of up to $10 \mathrm{kHz}$, to make a discrete excitation source. The total integration time of the PMT was $22 \mathrm{~ns}$, which was much smaller than the expected milliseconds lifetime region, and consequently showed a negligible impact of PMT in the measured milliseconds fluorescence lifetime of gold-ceria- NPs. In addition, both the rise and fall times of the optical pulses generated from the optical chopper at 10 were detected and found to be around $0.5 \mathrm{~ms}$.

\section{Results and Discussions}

Figure 1 a shows the fluorescence emissions of ceria NPs at around $530 \mathrm{~nm}$, under a violet excitation of $430 \mathrm{~nm}$, due to the formation of tri-valent trap states of cerium ions, which corresponded to $5 \mathrm{~d}-4 \mathrm{f}$ transition [5]. In addition, the plasmonic resonance emissions spectra of the used Au NPs is shown in Figure $1 b$, and both CdS/ZnS, and CdSe/ZnS QDs are shown together in Figure 1c [16,17]. The crosssection absorptions of plasmonic nanostructures show the wavelength resonance whether within the excitation wavelength of ceria at $430 \mathrm{~nm}$ or in the visible emissions at $530 \mathrm{~nm}$. Figure 2 shows the fluorescence emissions of different samples of ceria NPs embedded with plasmonic nanostructures, under a violet excitation of $430 \mathrm{~nm}$. All plasmonic-ceria NPs show an enhanced fluorescence visible emissions intensity compared to pure ceria NPs. That provides evidence of the optical coupling between the plasmonic resonance wavelength of the plasmonic nanostructures in general within either fluorescence excitation or the emissions spectrum region. However, the results show that Au NPs give a better emissions intensity when coupled with ceria fluorescence compared to quantum dots. 


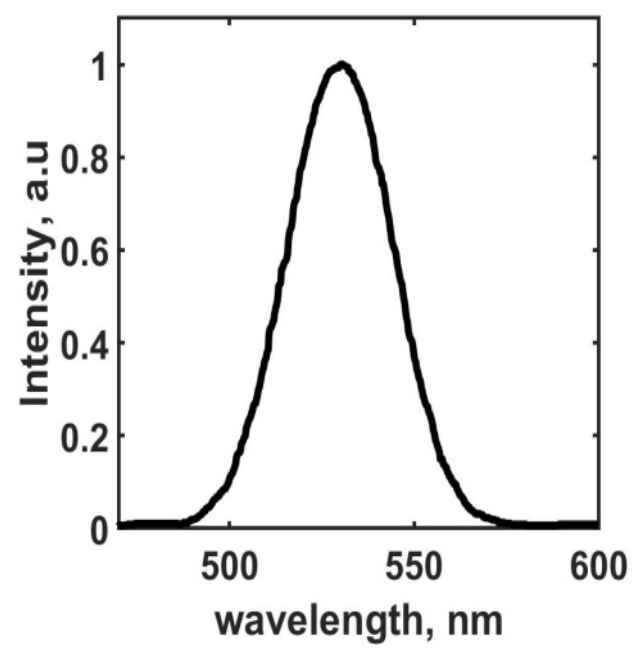

(a)

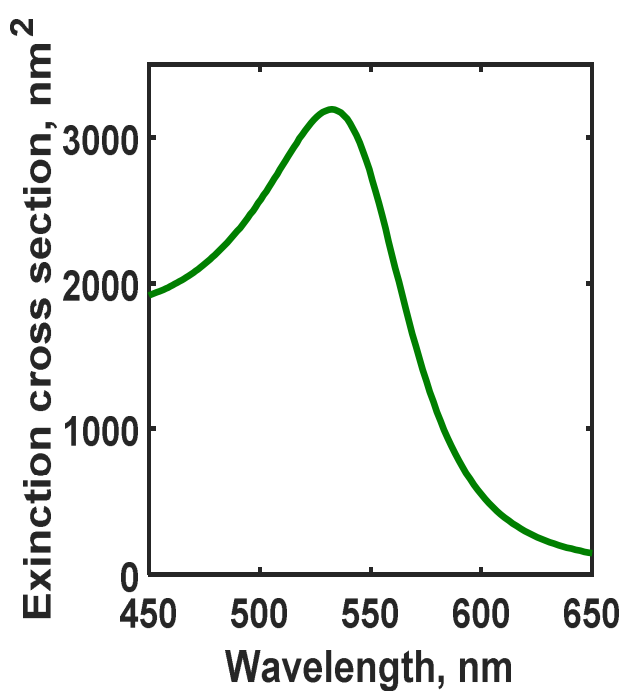

(b)

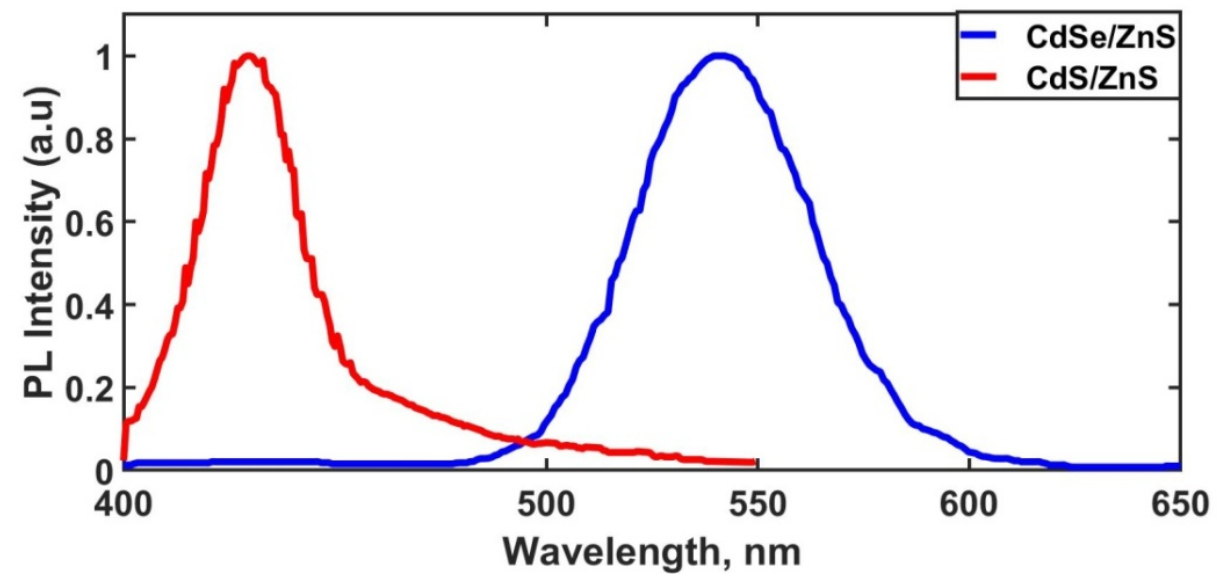

(c)

Figure 1. (a) Fluorescence emissions intensity of ceria nanoparticles with no added plasmonic nanostructures under $430 \mathrm{~nm}$ excitation, and absorption spectra for (b) gold nanoparticles with a plasmonic resonance wavelength of around $530 \mathrm{~nm},(\mathbf{c}) \mathrm{CdS} / \mathrm{ZnS}$ with a plasmonic resonance wavelength of around $430 \mathrm{~nm}$, and CdSe/ZnS quantum dots showing the plasmonic resonance wavelength at around $530 \mathrm{~nm}$.

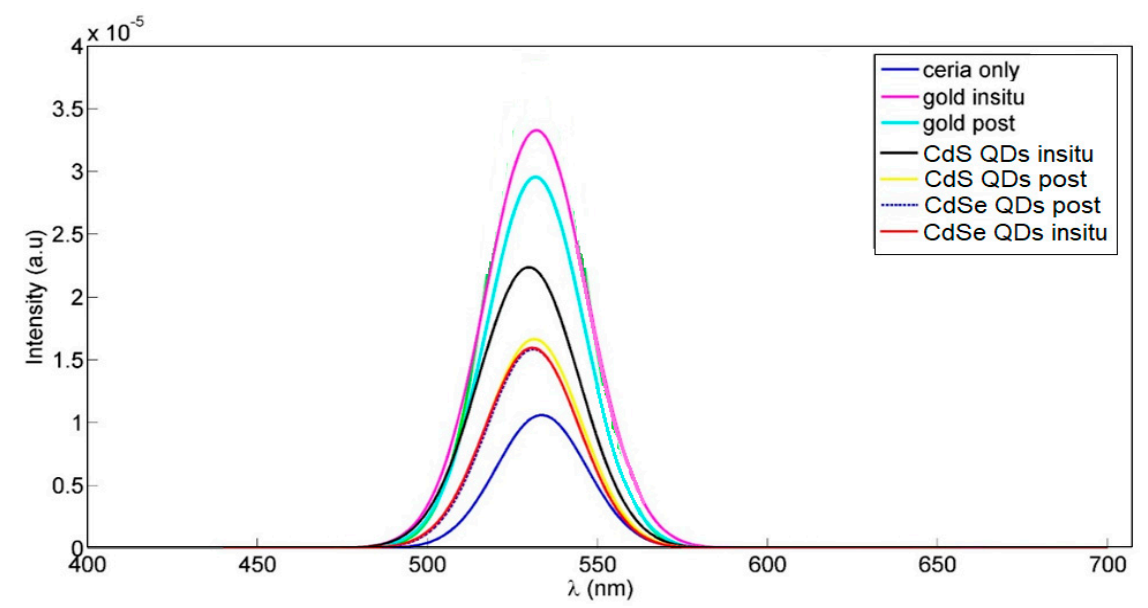

Figure 2. Fluorescence visible emissions of different plasmonic-embedded-ceria NPs. 
Focusing on Au NPs as shown in Figure 2, the in-situ added Au NPs of $530 \mathrm{~nm}$ plasmonic resonance wavelength show an enhanced fluorescence emissions peak, which was found to be higher for added AuNPs as a post-synthesis of the same wavelength. Hence, the in-situ case may indicate that an optical coupling between the plasmonic resonance wavelength of Au NPs with the fluorescence emissions intensity spectrum of ceria NPs has a higher probability of occurring compared to a coupling with Au NPs during post-treatment.

To study more features about the impact of added Au NPs on ceria NPs, Figure 3a shows the absorption spectroscopy analysis of ceria NPs, with and without added plasmonic nanostructures, along with the corresponding bandgap calculations in Figure 3b. Equation (1) illustrates the relationship between the allowed direct bandgap and linear region of the absorbance dispersion due to the Urbach tail phenomenon [18]

$$
\propto \mathrm{E}=\mathrm{A}(\mathrm{E}-\mathrm{Eg})^{1 / 2}
$$

where $\alpha$ is the optical absorbance, $\mathrm{A}$ is a material-dependent constant related to effective masses of electrons and holes, $\mathrm{E}$ is the absorbed photon energy, and Eg is the allowed direct bandgap. It can be observed that the in-situ added gold-ceria NPs gives the biggest reduction of ceria bandgap closer to $3 \mathrm{eV}$, compared to whether there are other plasmonic-added cases or pure ceria Au NPs. This provides evidence that the in-situ Au NPs with coupling impact of fluorescence emissions causes more tri-valent ionization states of cerium ions, associated with the formation of oxygen vacancy. In turn, this shifts the bandgap of non-stoichiometric $\mathrm{CeO}_{2-x}$ to the $3 \mathrm{eV}$ bandgap range. Therefore, that contributes to the results of highest fluorescence emissions intensity found in Figure 3, due to the formation of more tri-valent ions associated with more O-vacancies. Therefore, the added in-situ Au NPs can help to provide a better formation of tri-valent cerium ions, which correspond to a better probability of $5 \mathrm{~d}-4 \mathrm{f}$ transition and consequently leads to a higher fluorescence emissions intensity. Hence, the formation of more tri-valent cerium ions can be another possible factor for enhancing the fluorescence emissions, in addition to the possible coupling between ceria fluorescence emissions and plasmonic waves of $\mathrm{Au}$ NPs. Both factors together contribute to a higher fluorescence intensity peak. Figure 4a shows a transmission electron microscope (TEM) image of in-situ gold-ceria NPs, as an example. The mean diameter of ceria NPs is around $10 \mathrm{~nm}$, along with Au NPs with a $20 \mathrm{~nm}$ diameter. Figure $4 \mathrm{~b}$ shows an XRD pattern of gold-ceria NPs and the peaks of cerium oxide, with no peaks indicating the formation of gold oxide. By using the first stable plane of ceria crystalline structure (111), the ceria NPs' mean diameter was verified using the TEM image with mean diameter of $\sim 10 \mathrm{~nm}$.
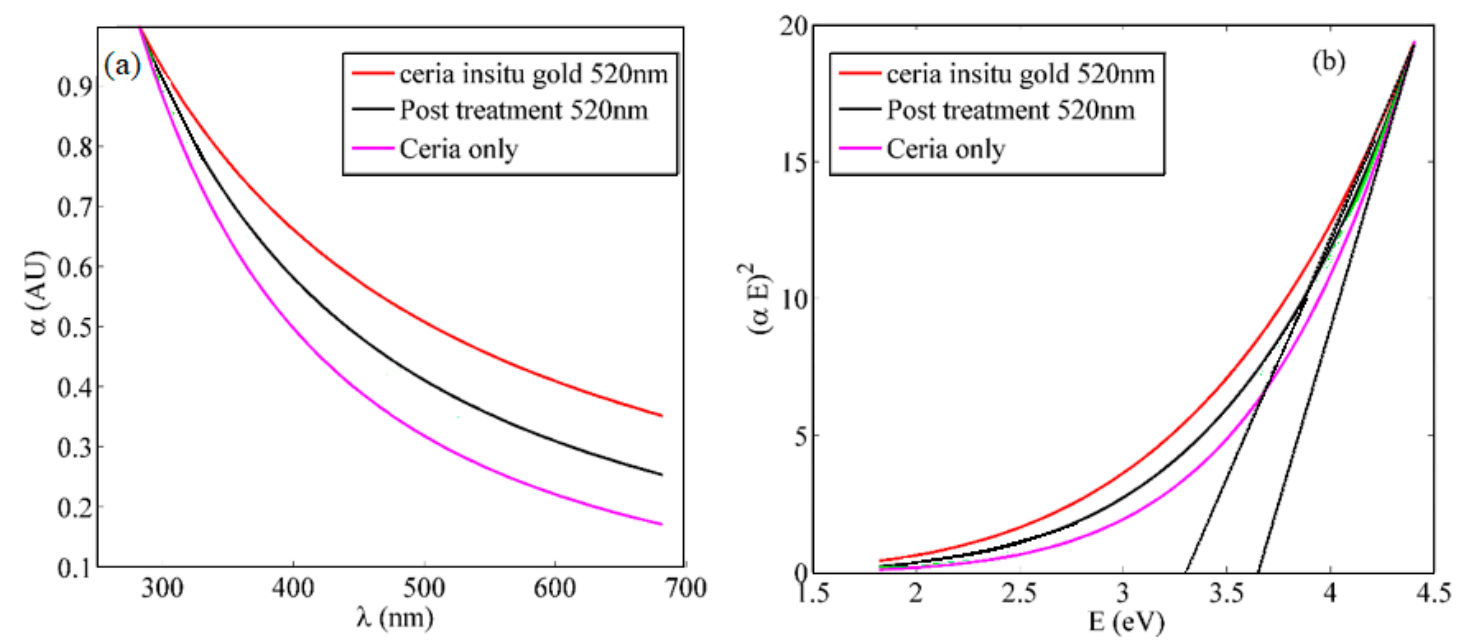

Figure 3. (a) Absorbance spectra, and (b) bandgap calculations of ceria NPs with and without added Au NPs. The added gold is in both cases of in-situ and post-synthesis. 

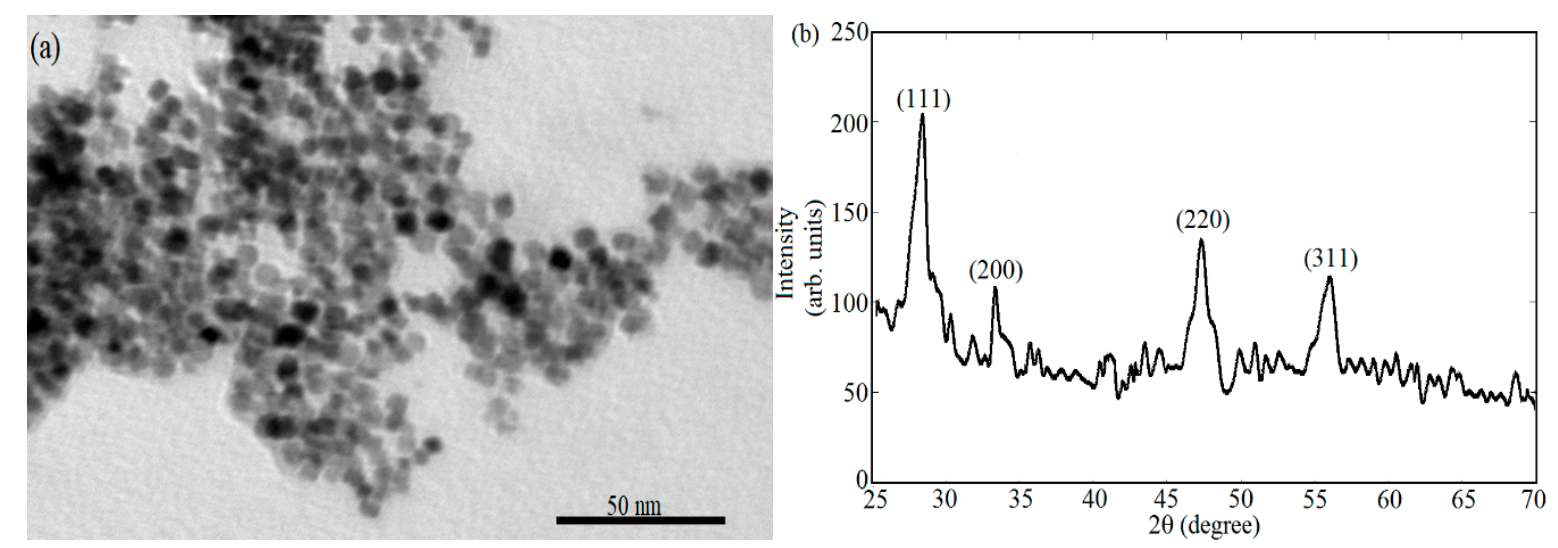

Figure 4. (a) TEM image of in-situ added gold-ceria nanoparticles, and (b) XRD pattern of gold-ceria NPs.

As an application, we have used our synthesized plasmonic-added-ceria NPs as an optical sensing material for the detection of metallic tiny particles in water, such as iron. The in-situ goldadded-ceria NPs was previously proved to have the highest fluorescence emissions intensity compared to post-synthesis gold-ceria, quantum dots-embedded-ceria, and pure ceria NPs. Therefore, we focus in this section on the application of in-situ gold-ceria NPs as the main sensitive host for the optical detection of tiny metallic iron particles. Figure 5 shows the fluorescence intensity quenching of in-situ gold-ceria NPs with an added increased concentration of iron tiny particles. It can be noticed that the fluorescence intensity was reduced, or quenched, to less than $40 \%$ of its initial emissions intensity with an added concentration of iron of up to $0.2 \mathrm{~g} / \mathrm{L}$. The fluorescence lifetime was also detected according to the variation of sensed tiny particle concentrations, as shown in Figure 6 . The fitted data of the fluorescence lifetime of in-situ gold-ceria NPs was found to be $0.42 \mathrm{~ms}, 0.30 \mathrm{~ms}$, and $0.23 \mathrm{~ms}$ for iron particles with concentrations of $0,0.03$, and $0.15 \mathrm{~g} / \mathrm{L}$, respectively. Therefore, the lifetime was decreased by up to approximately $55 \%$ of the initial lifetime at concentrations of iron quenchers of up to $0.15 \mathrm{~g} / \mathrm{L}$.

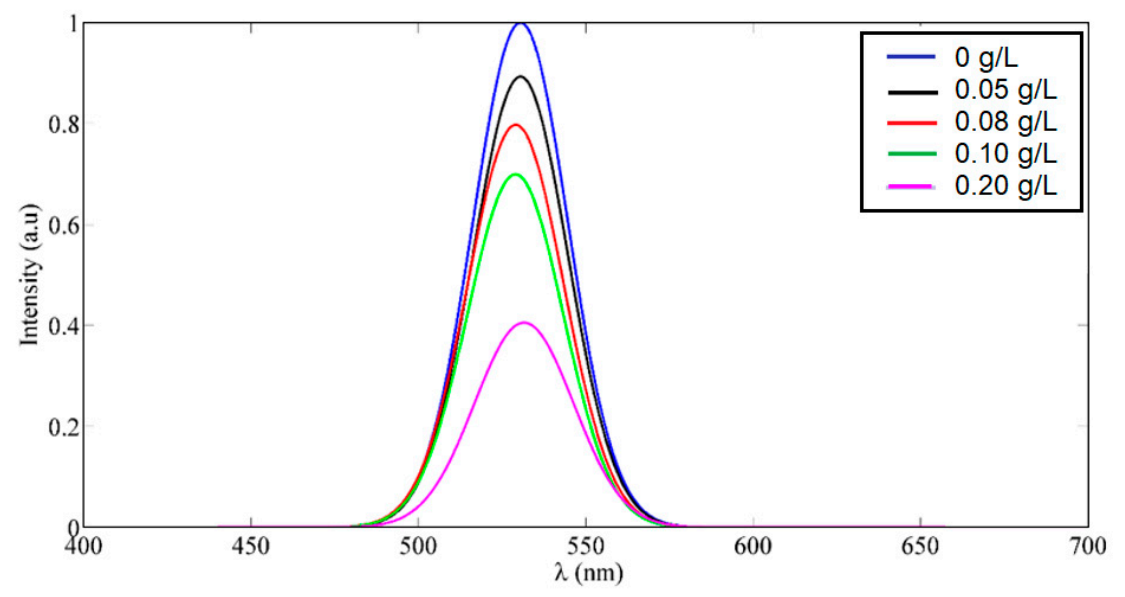

Figure 5. Fluorescence intensity quenching of in-situ gold-ceria NPs due to increasing iron particle concentrations "in $\mathrm{g} / \mathrm{L}$ ". 


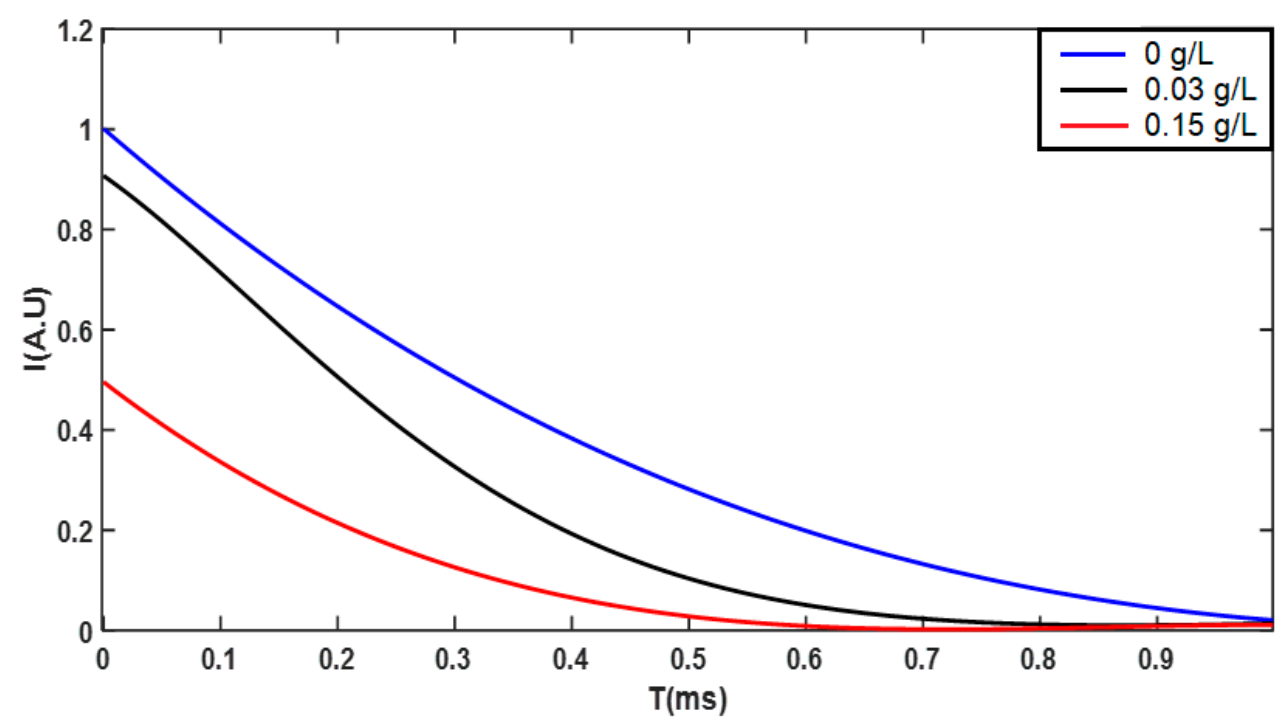

Figure 6. Fluorescence intensity lifetime variation of in-situ gold-ceria NPs due to increasing iron particle concentrations.

Based on the intensity change as shown in Figure 5, the relative change in the fluorescence emissions intensity at different iron concentrations is described in Figure 7, according to the Stern-Volmer relation [19]

$$
I_{0} / I=1+K_{S V}[Q]
$$

where both $I_{0}$ and $I$ are intensity peaks of fluorescence emissions in the absence and presence, respectively, of the iron tiny particles quenchers, $K_{S V}$ is the Stern-Volmer quenching constant which indicates the sensitivity of gold-ceria NPs in detecting the iron using a fluorescence quenching mechanism [14], and $[Q]$ is the iron quencher concentration. Figure 7 shows relatively linear behavior between the relative intensity and the quencher concentration at lower than $0.1 \mathrm{~g} / \mathrm{L}$, then the relation starts to be non-linear. In the linear region, the value of the calculated $K_{S V}$ is $8.6 \mathrm{M}^{-1}$ for in-situ gold-ceria NPs due to the iron quencher, compared to $3.6 \mathrm{M}^{-1}$ for ceria NPs only. While the optical sensitivity of gold-ceria nanoparticles to metallic iron tiny particles is higher than for pure ceria, it is also less linear.

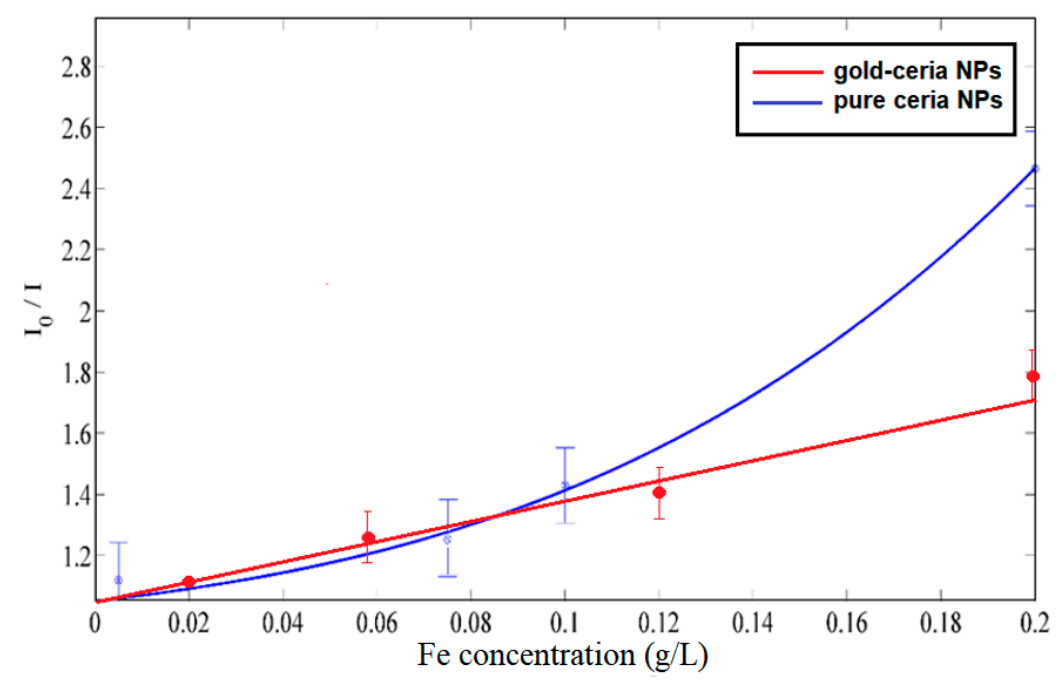

Figure 7. Relation between relative fluorescence intensity peak and variation of iron tiny particle concentrations for both gold-ceria nanoparticles and pure ceria nanoparticles "as a control sample". 
To verify whether the optical quenching mechanism is static or dynamic, the absorbance dispersion spectra of gold-ceria NPs was measured at different added iron tiny particles' concentrations, along with their corresponding bandgap calculations as shown in Figure 8. Both the absorbance and bandgap show a blue shift due to an incremental concentration of added metallic particles up to a $3.6 \mathrm{eV}$ bandgap at a $0.2 \mathrm{~g} / \mathrm{L}$ quencher concentration. That indicates the dominant static adsorption nature of tiny particles due to oxygen vacancies inside gold-ceria NPs. In dynamic quenching, the quenchers are reducing the fluorescence emissions intensity but without being part of the sensing material, which corresponds to no change in absorbance and bandgap. However, as shown in our work, the absorbance becomes changed, which corresponds to an adsorption of quenchers inside the sensing material.
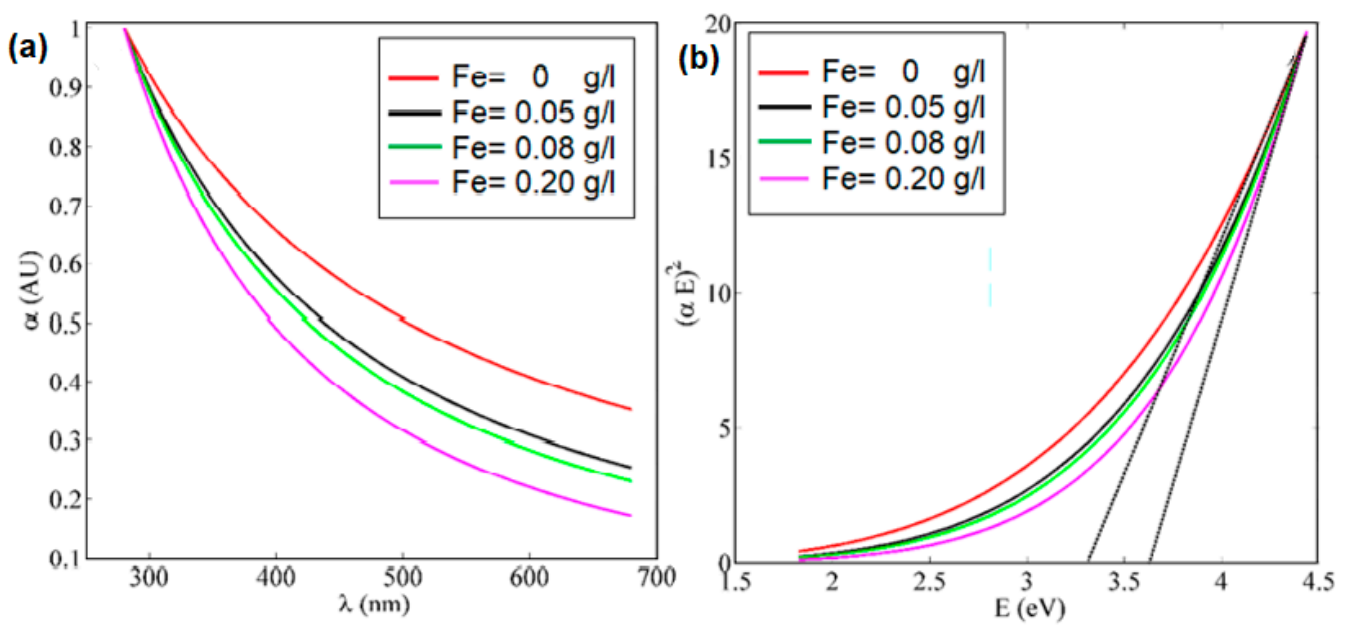

Figure 8. (a) Absorbance dispersion curve of in-situ gold-ceria NPs at different iron concentrations, and (b) the corresponding bandgap calculations.

\section{Conclusions}

In this work, the optical characteristics of ceria NPs have been studied due to the addition of different plasmonic nanostructures. We have selected Au NPs and QDs using plasmonic resonance wavelength selection through overlapping with both excitation and the emissions spectra of ceria NPs. It has been found that in-situ Au NPs provide the best enhancement of ceria fluorescence emissions, compared to post-added gold or added QDs, regardless of whether they are in-situ or post-added. Based on absorbance and bandgap analysis, Au NPs contribute to improving the formation of tri-valent cerium ions with associated O-vacancies, in addition to the coupling between the plasmonic resonance of gold and fluorescence emissions of ceria. Based on their fluorescence enhancement, ceria-gold NPs are used as an optical sensor for iron tiny particles in aqueous media through a fluorescence quenching mechanism with a Stern-Volmer constant of $8.6 \mathrm{M}^{-1}$, which is 2.4 times more effective than pure ceria nanoparticles. The quenching mechanism was found to be static based on absorbance dispersion measurements and corresponding bandgap calculations, which shows the change in the bandgap of gold-ceria NPs due to being adsorbed iron tiny particles.

Author Contributions: Conceptualization, N.S. and I.K.; Methodology, N.S., E.S. and I.K.; Resources, N.S.; Data Curation, E.S.; Writing-Original Draft Preparation, N.S.; Writing-Review \& Editing, N.S. and I.K.; Visualization, E.S.; Supervision, N.S. and I.K.; Project Administration, N.S.; Funding Acquisition, N.S. All authors have read and agreed to the published version of the manuscript.

Funding: The project was funded "partially" by Kuwait Foundation for the Advancement of Sciences under project code: PR17-15EE-01.

Acknowledgments: The authors would like to thank Mohamed Salah for his support in initiating the optical fluorescence setup at Kuwait College of Science and Technology (KCST). Also, the authors would like to thank KCST administration for logistic support along with offering some chemical facilities inside Lab Nanotechnology and Photonics (LNP) at KCST. 
Conflicts of Interest: The authors declare no conflict of interest. The funders had no role in the design of the study; in the collection, analyses, or interpretation of data; in the writing of the manuscript, or in the decision to publish the results.

\section{References}

1. Quaranta, M.; Borisov, S.M.; Klimant, I. Indicators for optical oxygen sensors. Bioanal. Rev. 2012, 2, 115-157. [CrossRef] [PubMed]

2. Skala, T.; Tsud, N.; Prince, K.C.; Matolin, V. Formation of alumina-ceria mixed oxide in model systems. Appl. Surf. Sci. 2011, 257, 3682-3687. [CrossRef]

3. Shehata, N.; Meehan, K.; Hassounah, I.; Hudait, M.; Jain, N.; Clavel, M.; Elhelw, S.; Madi, N. Reduced erbium-doped ceria nanoparticles: One nano-host applicable for simultaneous optical down- and up-conversions. Nanoscale Res. Lett. 2014, 9, 231. [CrossRef] [PubMed]

4. Chorvat, D.; Chorvatova, A. Multi-wavelength fluorescence lifetime spectroscopy: A new approach to the study of endogenous fluorescence in living cells and tissues. Lasers Phys. Lett. 2009, 6, 175-193. [CrossRef]

5. Shehata, N.; Meehan, K.; Jain, N. Control of oxygen vacancies and $\mathrm{Ce}^{+3}$ concentrations in doped ceria nanoparticles via the selection of lanthanide element. J. Nanoparticle Res. 2012, 14, 1173-1183. [CrossRef]

6. Feng, N.; Xie, J.; Zhang, D. Synthesis, characterization, photophysical and oxygen-sensing properties of a novel europium(III) complex. Spectrochim. Part A 2010, 77, 292-296. [CrossRef] [PubMed]

7. Rzigalinski, B.A.; Meehan, K.; Davis, R.M.; Xu, Y.; Miles, W.C.; Cohen, C.A. Radical nanomedicine. Nanomedicine 2006, 1, 399-412. [CrossRef] [PubMed]

8. Shehata, N.; Azab, M.; Kandas, I.; Meehan, K. Nano-Enriched and Autonomous Sensing Framework for Dissolved Oxygen. Sensors 2015, 15, 20193-20203. [CrossRef] [PubMed]

9. Garcia-Garcia, F.J.; Yubero, F.; González-Elipe, A.; Lambert, R. Microstructural Engineering and Use of Efficient Poison Resistant Au-doped Ni-GDC Ultrathin Anodes in Methane-fed Solid Oxide Fuel Cells. Int. J. Hydrogen Energy 2018, 43, 885-893. [CrossRef]

10. Wang, J.; Xiao, X.; Liu, Y.; Pan, K.; Pang, H.; Wei, S. The application of $\mathrm{CeO}_{2}$-based materials in electrocatalysis. J. Mater. Chem. A 2019, 7, 17675-17702. [CrossRef]

11. Gao, Y.; Li, X.; Wu, H.; Meng, S.; Fan, X.; Huang, M.; Guo, Q.; Tung, C.; Wu, L. Exceptional Catalytic Nature of Quantum Dots for Photocatalytic Hydrogen Evolution without External Cocatalysts. Adv. Funct. Mater. 2018, 28, 1801769. [CrossRef]

12. Pawlis, S.; Berth, G.; Wiedemeier, V.; Schmidt, L.; Zrenner, A.; Warneck, H.J. Oxygen sensing by fluorescence quenching of [Cu(btmgp)I]. J. Luminscence 2010, 130, 1958-1962. [CrossRef]

13. Chu, C.; Lo, Y. A plastic optical fiber sensor for the dual sensing of temperature and oxygen. IEEE Photonics Technol. Lett. 2008, 20, 63-65. [CrossRef]

14. Iosin, M.; Canpean, V.; Astilean, S. Spectroscopic studies on pH and thermally induced conformational changes of bovine serum albumin adsorbed ontogold nanoparticles. J. Photochem. Photobiol. 2011, 217, 395-401. [CrossRef]

15. Chen, H.; Chang, H. Homogeneous precipitation of cerium dioxide nanoparticles in alcohol/water mixed solvents. Colloids Surf. A Physicochem. Eng. Asp. 2004, 242, 61-69. [CrossRef]

16. Galloway, J.F.; Park, J.; Lee, K.H.; Wirtz, D.; Searson, P.C. Exploiting Nucleation and Growth in the Synthesis and Electrical Passivation of CdSe Quantum Dot. Sci. Adv. Mater. 2009, 1, 93-100. [CrossRef]

17. Sabir, N.; Qayyum, W.; Hussain, S.Z.; Hussain, I.; Amin, F. Photoluminescence properties of Co and Ni co-doped CdS/ZnS core/shell nanoparticles. In Proceedings of the Colloidal Nanoparticles for Biomedical Applications XIII, San Francisco, CA, USA, 27 January-1 February 2018.

18. Pankove, J. Optical Processes in Semiconductors, 1st ed.; Dover Publications Inc.: New York, NY, USA, 1971.

19. Jiang, Z.; Yu, X.; Zhai, S.; Hao, Y. Ratiometric Dissolved Oxygen Sensors Based on Ruthenium Complex Doped with Silver Nanoparticles. Sensors 2017, 17, 548. [CrossRef] [PubMed]

(C) 2020 by the authors. Licensee MDPI, Basel, Switzerland. This article is an open access article distributed under the terms and conditions of the Creative Commons Attribution (CC BY) license (http://creativecommons.org/licenses/by/4.0/). 